\title{
QUITTE OU DOUBLE ? ROBBE-GRILLET ET LE MYTHE DE L'IDENTITÉ
}

\section{CÉCILE VOISSET-VEYSSEYRE UNIVERSITÉ PARIS XII}

pa.veysseyre@orange.fr

Article received on 31.01.2011

Accepted on 29.05.2011

\section{RÉSUMÉ}

Une lecture du texte d'Alain Robbe-Grillet met à jour la force d'une écriture qui démystifie c'est-à-dire qui évacue le contenu représentatif. Sur le mode cinématographique, s'élabore une pratique littéraire qui fait sienne la coupure comme ressort narratif et pour laquelle, dans le cadre d'un nouveau roman qui problématise la perception de soi, le montage définit tout l'espace du jeu; si l'auteur est mort, l'autobiographie aussi. L'écrivain - le scripteur - expérimente une forme romanesque dont la modernité tranche avec la linéarité du récit traditionnel; non seulement le moi ne s'y invente pas, mais il s'y ruine de manière emblématique sous la figure du miroir brisé. La traversée du fantasme, celui du double c'est-à-dire de l'un et du multiple, conduit à l'analyse textuelle d'une identité dont le mythe se porte garant.

MOTS-CLÉ

Double, identité, imaginaire mythique, lignes, écriture.

\section{DOUBLE OR QUITS? ROBBE-GRILLET AND THE MYTH OF IDENTITY}

\section{ABSTRACT}

A reading of Alain Robbe-Grillet's text brings up to date the force of a demystifying writing, i.e., which empties the representative content. Under the influence of cinema, a new literary practice emerges, which claims the cutting as a narrative resource of its own in the context of a new novel which challenges self-perception; the ultimate montage defines thus the whole gaming space. If the author is dead, so is autobiography. The writer - the scripter - experiences a modern novelistic form in sharp contrast with linear, traditional narratives. Not only has the self ceased to invent itself, but gets emblematically destroyed in the figure of the shattered mirror. The wandering ghost - that of the double, of the one and the multiple - leads to the textual analysis of an identity which the myth assures.

\section{KEYWORDS}

Double-self, identity, mythic imagination, lines, writing. 
« Vous croyez tous être toujours dans le domaine psychologique, et que la psyché est une espèce de double, et de propriété, de ce quelque chose que vous voyez ${ }^{1}$

«Alors mon double est là ce soir? Tu devrais me le montrer ! »²

« Qu'est-ce que c'est, moi ? Et qu'est-ce que je fais là ? »3

\section{INTRODUCTION: QUITTE OU DOUBLE?}

Ce qui distingue le mythe moderne du mythe antique, c'est que celui-ci dit l'origine et celui-là le moi et sa morale: « les mythes grecs ne sont pas moïques »4. La latinité aurait alors pu supplanter une grécité trop attachée à sa philosophie telle qu'elle vise à rationaliser d'anciens récits selon une opposition reçue entre mythos et logos. En tant que genre narratif, le mythe participe certainement d'une forme de la croyance sous l'espèce d'une parole idéologique c'est-à-dire déployée dans l'espace de la représentation; il parle à l'imagination collective.

Dans Différence et répétition, Gilles Deleuze donnait à repenser la pratique philosophique c'est-à-dire l'écriture de cette antique discipline dont la raison aurait fait son territoire voire son terrain de chasse: « Un livre de philosophie doit être pour une part une espèce très particulière de roman policier, pour une autre part une sorte de sciencefiction. ${ }^{5}$ À le prendre au mot, on peut considérer que les romans d'Alain Robbe-Grillet sont des romans philosophiques et rappeler que ses fictions répondent à sa propre théorisation du roman; on le peut compte tenu de ce que le philosophe écrivait au sujet de la pensée moderne telle qu'elle est née « de la faillite de la représentation, comme de la perte des identités ${ }^{6}$. Il resterait à savoir si le mythe, cette parole qui ne dit rien qui vaille parce qu'elle ne parle que du même, doit être défait par la pratique littéraire ignorant la pratique philosophique comme son démon; cela reviendrait à savoir si le sujet, défini par son image unifiée dans le miroir, n'est pas en voie de disparition selon le texte robbegrilletien qui s'essaie à le gommer. Il n'est pas fortuit que le thème de l'un et par conséquent du double - du triple, du quadruple, du septuple, de l'octuple, etc. - c'est-àdire du multiple, thème qui marque aussi le texte deleuzien, insiste dans celui d'un roman enfin débarrassé des scories représentatives et aéré par les reflets de miroirs éblouissant sa lecture. L'écriture cinématographique les réunit alors non par la question de l'image telle que la développent des études contemporaines et paresseuses où le texte est soustrait à sa complexité et réduit au format d'une pensée arrêtée, mais selon l'objectif et la question du

\footnotetext{
${ }^{1} \mathrm{~J}$. Lacan, Le Séminaire. L. II, Paris: Seuil, 1978, p. 130.

${ }^{2}$ A. Robbe-Grillet, Projet pour une révolution à New York, Paris: Minuit, 1970, p. 50.

${ }^{3}$ A. Robbe-Grillet, Angélique ou l'enchantement, Paris: Minuit, 1987, p. 69.

${ }^{4}$ J. Lacan, op. cit., p. 306.

${ }^{5}$ G. Deleuze, Différence et répétition, Paris: PUF, 1989, p. 3.

${ }^{6}$ G. Deleuze, Différence et répétition, p. 1.
} 
montage. Ainsi le scripteur, plutôt que l'écrivain, s'expliquait-il: « J e me mis donc à écrire, seul, non pas une "histoire", mais directement ce que l'on appelle un découpage »7. Le ciné-roman, qui est « description ${ }^{8}{ }^{8}$, s'emploie à démonter cette mer d'images que serait la mythologie comme forme renvoyée de l'inconscient; le nouveau roman conteste cette parole une, encadrée, que redistribuent les « mythes modernes ${ }^{9}$. Hors des cadres rigides, le projet de Robbe-Grillet s'inscrit dans une optique où ce qui prime est ce qui est vu du dehors c'est-à-dire selon le point d'où cela est jalousement pris; il y a distanciation dans le nouveau texte. L'image n'est qu'un instantané, qu'une entaille dans le vif, pas un cliché photographique; elle n'est pas enchaînement raisonné qui déroulerait une linéarité lisse, emportant l'adhésion et l'identification du lecteur. Les coup(ur)es incessantes font que tout renvoie à tout et que ponctuer signifie non pas arrêter mais ne pas mettre de points finaux, comme si le récit devait avoir une fois pour toutes un début - un milieu - et une fin: une écriture soigneuse « se termine, au milieu d'une ligne par une phrase interrompue, sans signe de ponctuation après le dernier mot. »10 L'image est démythifiée, déconnectée d'un ensemble qui aurait dû faire sens et conspirer à l'idée de raconter une histoire de fond qui précéderait à sa forme. Dans ce contexte, le contestataire retire toute pertinence à la question du Sujet c'est-à-dire d'un sujet en majesté qui présiderait au bon déroulement de la parole récitante; il ricane volontiers sur « notre sujet multinominal et volontiers pseudonyme »11.

Le soupçon est jeté, la recherche est en cours; à tâtons dans le gris du brouillon, Robbe-Grillet s'écrie: « J'ai avancé cette supposition à tout hasard, pour voir, à votre réaction, si vous aviez quelque chose à nous apprendre sur un sujet à peine dégrossi, en pleine mouvance narrative... »12 Or, celui qui manifeste déclare: « il est faux que tout soit double »13. Serait-ce qu'il faille se passer de l'Un, de l'unité? La philosophie de la dernière modernité passerait-elle par ce nouveau roman dont le texte s'écrit sur les restes d'une mythologie grecque dont « Corinthe » - écrit à l'occasion avec un « i » ${ }^{14}$ grec - est le nom

${ }^{7}$ A. Robbe-Grillet, Introduction à L'année dernière à Marienbad, Paris: Minuit, 1961, p. 10.

8 A. Robbe-Grillet, Notes préliminaires à L'Immortelle, Paris: Minuit, 1963, p. 7. L'introduction aux Glissements progressifs du plaisir (Paris: Minuit, 1974, p. 9) rappelle ces « descriptions » qui sont les coupures successives et constitutives du scénario; or, le double paraît comme l'un des principes de cette méthode de rupture qui soustrait l'esprit à toute mythification (p. 12): « trop de fragmentations, de ponctuations, de plans isolés (comme situés entre parenthèses), de répétitions ou de dédoublements, y figurent en évidence pour que le sens anecdotique des séquences ou bouts de séquence qui demeurent intactes entre deux intrusions, deux infractions, des coupures, n'en perde pas un peu de sa belle assurance. »

9 A. Robbe-Grillet, Avant propos à Projet pour une révolution à New York.

10 A. Robbe-Grillet, Instantanés, Paris: Minuit, 1962, p. 56.

${ }^{11}$ A. Robbe-Grillet, La Reprise, Paris: Minuit, 2001, p. 65.

12 A. Robbe-Grillet, La Reprise, p. 247.

${ }^{13}$ A. Robbe-Grillet, Pour un nouveau roman, Paris: Minuit, 1963, p. 67.

${ }^{14}$ A. Robbe-Grillet, Les Derniers jours de Corinthe, p. 87: « ([...] une déformation hellénique de l'ancienne appellation qui désignait notre fief médiéval: Quimper-Corentin) ». Le «i » grec revient dans l'un des premiers livres (Le Voyeur, Paris: Minuit, 1955, p. 55 et p. 133). 
rimant avec « labyrinthe »? Sous les traits improbables de son antihéros, l'écrivant n'y allait pas par quatre chemins en empruntant la voie de l'anonymat pour son promeneur. La dés-écriture du moi pousse à réfléchir sur une pseudo-narration de nous-mêmes, quitte à laisser place à une écriture impossible du soi pris pour objet de lui-même; un mot dit cette narration fictionnelle et non véridique de soi: auto-mytho-graphie. La nouvelle parole démantèle par là l'ancienne idéologie répondant d'un prétendu réalisme. Avec elle, le contenu représentatif est mort; le jeu sur le signifiant congédie le signifié et rend de ce fait inefficace le principe mythique d'une clôture de sens. Que l'on se rappelle pour lors ce qu'écrivait une historienne des représentations, spécialiste de la pólis - « Les mythes [...] disent à la cité son identité » 15 - et l'on entendra pourquoi le texte robbe-grilletien - où le motif de la ville est cousu à celui du double comme au moi - met fin à cette identité dont on peut penser qu'elle est le mythe des mythes.

Le fil du double c'est-à-dire du reproductible, selon le principe de la répétition et de la variation, oriente la lecture et conduit au plaisir de son texte, selon le principe jubilatoire qui consiste à ne pas s'arrêter c'est-à-dire à ne point se centrer. Le texte de Robbe-Grillet joue. Par pur plaisir, il joue à dérouter; il joue de la liberté de couper le récit et d'en découper les séquences. Une première séance suit ce fil qui le mène à traverser « le mythe de l'intériorité ${ }^{16}$, ce mythe de la profondeur dont il ne voulait plus entendre parler; elle tient compte de ce que Deleuze écrivait dans sa défense du néo-réalisme: «Ce que nous appelons normalité, c'est l'existence des centres: centres de révolution même, d'équilibre des forces, de gravité des mobiles, et d'observation pour un spectateur capable de connaître ou de percevoir le mobile, et d'assigner le mouvement. »17 L'acentralité narrative fait dorénavant de l'errance non seulement un principe sublunaire assumé mais aussi une liberté dont la marge de manœuvre tient à ce double perpétuel du texte robbe-grilletien. De la « double porte fermée ${ }^{18}$ ou de 《la double porte vitrée ${ }^{19}$, on passe au double du moi qu'on entend habituellement comme alter ego c'est-à-dire comme double de soi; c'est au pluriel - doubles - qu'il faut l'entendre, tel cet inconnu marchant sur la même plage que le narrateur et longeant comme lui la mer: « Il y avait deux personnes, maintenant, en face de lui ${ }^{20}$. Ainsi, la lecture passe sans transition à une seconde séance; elle va de l'un à l'autre sans que cela revienne au même c'est-à-dire qu'elle va constamment de l'un au deux

${ }^{15}$ N. Loraux, Né de la terre. Mythe et politique à Athènes, Paris: Seuil, 1996, p. 58.

${ }^{16}$ Nous reprenons cette expression à J. Bouveresse qui entreprenait une critique du sujet au regard de la scène philosophique (Le mythe de lintériorité, Paris: Minuit, 1987, Introduction, p. 57): « Il y a donc bien un "mythe de l'intériorité", en ce sens qu'il y a une conception mythique du mode de fonctionnement du langage psychologique, et non pas au sens où les états et les processus internes qu'il est censé décrire seraient purement mythiques. » Nous l'entendons au sujet du mythe défini comme parole puissante à laquelle on croit, pouvoir auquel on se soumet.

${ }^{17}$ G. Deleuze, Cinéma 2, Paris: Minuit, 1985, p. 53.

${ }^{18}$ A. Robbe-Grillet, Instantanés, p. 89. On trouve également les idées et les images de double clef, de double fond, de double série ou de double tour, de double alignement, etc.

${ }^{19}$ A. Robbe-Grillet, La maison de rendez-vous, Paris: Minuit, 1965, p. 91.

${ }^{20}$ A. Robbe-Grillet, Un Régicide, Paris: Minuit, 1978, p. 165. 
et plus s'il le faut. C'est que le fantasme du double organise la désagrégation de toute identité.

\section{LE FANTASME DU DOUBLE}

On a bien sûr relevé « l'affirmation renouvelée du thème du double, sous diverses variations, comme mélodie même du texte » 21 de Robbe-Grillet; mais on l'a fait dans un sens contraire à toutes les directions que ce dernier a données au parcours de son lecteur égaré voire dévoyé. Ce thème déjoue toute tentative de centralité ou d'égocentricité par la mise en avant du plan de perception; telle vaut cette fin de la narration labyrinthique qui nous tient en haleine au sujet d'un énigmatique et insignifiant soldat blessé: « ..., et toute la ville derrière moi. »22. Ce dernier, on ne sait justement pas ce qu'il est; on sait juste qu'il est doublé et dépassé par toutes sortes de choses. Bien sûr, c'est son double que ce soidisant sujet objectivé, privilégié; pourtant, on nous a prévenu: « Définir la nature du moi entraîne très loin. »23 Par les sentiers robbe-grilletiens, se fraye l'idée que cette formation imaginaire s'illustre non par le mythe de Narcisse mais par celui d'Edipe. Ainsi la vue vat-elle de pair avec le non-vu ou le non entièrement vu et le double avec le trouble dans un texte anti-œedipien c'est-à-dire qui refuse le vieux mythe fondateur de notre identité occidentale en épousant pour le jeu les qualités de l'aveugle et en décrédibilisant par avance toute idée de double vue c'est-à-dire de don qui ramènerait le lecteur et l'auteur au divin: « ma cécité volontaire en serait une sorte de métaphore, ou d'image objective, ou de redoublement... » ${ }^{24}$ Le double n'a rien de narcissique dans le texte où l'idée du moi double celle de sujet; là aussi « ce sujet, en somme, est personne » ${ }^{25}$. Le double se dit là de tout objet, pas seulement du soi pris pour tel. Écrivant un moi mal réfléchi, ce texte fait éclater l'opposition du sujet et de l'objet; il inscrit seulement la récurrence comme motif échappant à l'identitaire c'est-à-dire à l'autoritaire de l'un dont - d'où - suit le deux. Par l'image du voyeur, le regard se fait avancée c'est-à-dire qu'il devient en vue; la vue n'est jamais donnée immédiate ni délimitée par un cadre représentatif aux coordonnées spatiotemporelles stables.

Cousu au motif du miroir, celui du double met en abyme cette âme que la Philosophie s'imagine comme l'ensemble des phénomènes psychiques qu'enveloppe le corps. Chez

${ }^{21}$ R.-M. Allemand, Duplications et duplicité dans les “Romanesques” d'Alain Robbe-Grillet, Paris: Lettres modernes, 1991, p. 59.

${ }^{22}$ A. Robbe-Grillet, Dans le labyrinthe, Paris: Minuit, 1959, p. 221.

${ }^{23} \mathrm{~J}$. Lacan, Le Séminaire. Livre II, p. 11.

${ }^{24}$ A. Robbe-Grillet, Djinn, Paris: Minuit, 1981, p. 102. Dans ce livre où le deux thématise la gémellité, la qualité d'aveugle (p. 62) et les troubles aigus de vue (p. 145), est visé l'héritage mythique d'Édipe à Colo(g)ne. Dans son entretien avec B. Peeters, A. Robbe-Grillet revient sur l'importance du texte sophocléen (notamment Edipe-Roi) dans son texte en général.

${ }^{25} \mathrm{~J}$. Lacan, Le Séminaire. Livre II, p. 72. Le fameux sujet n'est même pas « Personne » dans les Romanesques où figure « Ulysse » (nom du grand-père Robbe, instituteur à la retraite), sauf à s'entendre sous le nom de Nemo où il est effectivement convoqué. 
Robbe-Grillet, la psyché fait signe vers cette grande glace mobile qui fait voir en sa totalité quiconque s'y regarde en pied et à plusieurs reprises tant l'image en est dépolie; « le miroir ovale ${ }^{26}$ est ce haut miroir qui renvoie sa surface lisse sans rendre sa face présentable. Des éclats sautent aux yeux de la lecture, impossible qu'il est de voir en tout et pour tout c'est-à-dire d'envisager et d'embrasser du regard autrement que par oblique ou de biais: « en profil perdu »27. C'est le cas dans « cette autobiographie »28 où le mythe du double s'écrit en l'espèce comme ressac, reflux de l'océan, retour d'éclats de verres, que les brisures de la mer font figurer comme en un « miroir fantôme » ${ }^{29}$; ce « vif miroir éphémère »30 rend des images intermittentes. Ainsi le double miroite-t-il dans un texte qui met en exergue le regard acéré, lamellisé; ainsi l'eau-miroir rend-elle une image troublée ou ridée, du méconnaissable: « dans l'eau du port l'image renversée de l'ensemble et, à la surface, toujours dans le même jeu de parallèles... »31 Les Romanesques désigne cette trilogie autobiographique qui met à l'écrit ce personnage curieux qu'est Henri de Corinthe - le comte Henri, le lieutenant-colonel au nom décliné en allemand, le fringuant capitaine à la lignée germanique, le jeune et blême chevalier montant un coursier blanc - c'est-àdire « le protagoniste à double face »32. Avec ce héros « négatif »33, Robbe-Grillet détaille son projet de détruire les normes traditionnelles d'expression: «Quant au moi, de tout temps haïssable, il prépare ici sans aucun doute une rentrée en scène encore plus frivole: celle du biographisme. » ${ }^{34}$ C'est donc Pascal qui est ici appelé pour en finir avec l'illusion d'être tout, ou plutôt d'être un tout; ce sont ses Pensées qui ordonnent la direction de cet athée refusant autant le sacre de l'écrivain que celui du sujet et ne croyant nullement « qu'il ne faut aimer que Dieu, et ne haïr que soi-même »35. Le tissu du texte révolutionnaire ne prêtera plus à d'autre lutte qu'à celle menée contre le pouvoir narratif: « J'affirme récuser l'entreprise autobiographique, où l'on prétend rassembler toute une existence vécue [...] en un volume clos, sans manques et sans bavures »36. Le double mis à

26 A. Robbe-Grillet, La Jalousie, Paris: Minuit, 1957, p. 142. On retrouve l'image dans Les Derniers jours de Corinthe (p. 57: un « pesant miroir ovale »), dans Le Voyeur (p. 77: « la grande glace ovale ») et dans Topologie d'une cité fantôme (Paris: Minuit, 1976, p. 10 et p. 65).

27 A. Robbe-Grillet, La Jalousie, p. 184. Voir aussi les Instantanés (p. 52, 54 et 106), LImmortelle (pp. 156, 83, 110 et 126) et Projet pour une révolution à New York (p. 9). Ce qui ne peut être vu qu'obliquement dit l'insaisissable ou le fuyant (Le Miroir qui revient, p. 12): « Mais à présent que je me résous [...] à me regarder de côté, ce point de vue inattendu me libère soudain de mes anciennes protections et réticences. »

28 A. Robbe-Grillet, Le Miroir qui revient, p. 9.

${ }^{29}$ A. Robbe-Grillet, Le Miroir qui revient, p. 102. La Topologie d’une cité fantôme parle de « la surface trouble du miroir » (p. 12) et de « miroir liquide» (p. 79).

30 A. Robbe-Grillet, Les Derniers jours de Corinthe, p. 8.

${ }^{31}$ A. Robbe-Grillet, Le Voyeur, p. 13.

${ }^{32}$ A. Robbe-Grillet, Le Miroir qui revient, p. 74.

33 A. Robbe-Grillet, Angélique ou l'enchantement, p. 155.

${ }^{34}$ A. Robbe-Grillet, Le Miroir qui revient, p. 10.

35 B. Pascal, Pensées, Texte établi par L. Brunschvicg, GF, 1976, p. 129. On distinguera bien sûr le je du moi.

${ }^{36}$ A. Robbe-Grillet, Le Miroir qui revient, p. 58. 
l'honneur de l'écriture subversive permet de jouer et de se déjouer, de s'amuser à doubler et dédoubler, à compliquer, à dupliquer: à répliquer. Que vole en éclats ou en bris le miroir coupant que le texte a prévu fêlé et en partie cassé, alors même qu'on lit: « j'ai vu mon double pour la première fois. ${ }^{37} \mathrm{Ce}$ jeu prévaut au détriment du pacte censé définir un genre désormais perverti: « Peut-on nommer cela comme on parle de Nouveau roman, une Nouvelle Autobiographie »38 ? Certainement, à condition de préciser que Robbe-Grillet écrit contre le mythe d'une totalité que réfléchit en partie celui mortifère de l'androgyne c'est-à-dire du côte à côte, du côté, du flanc, du coteau, de la moitié, de la coupe. L'écriture par dissémination se charge maintenant d'« instituer le plan comme unité narrative, et non plus la séquence. »39 Le bris - de glace - figure le fragmentaire; le débris et la lumière font sens simultanément, tout commel'image de la vague caresse l'idée du flou.

Le regard découpe son objet, la jalousie n'est pas que sentiment; écrire c'est tirer des traits, tracer un axe perceptif selon le volet plus ou moins ensoleillé mais ce n'est pas dessiner c'est-à-dire épouser un contour aux formes figées. L'importance des formes géométriques dans le texte robbe-grilletien transforme une réflexion d'écriture en réflexion politique par où la frontière se lit pourquoi pas à la lumière du texte lacanien disant l'inconscient comme oubli: «Le redoutable inconnu au-delà de la ligne ${ }^{40}$. La duplication incessante des miroirs dit et redit le double comme fausseté ou mystification. Si tout renvoie à tout ou si tout est dans tout, alors que reste-t-il en effet à dire du Je - de nous - et du double? L'aristotélisme n'a plus lieu d'être: « Notre propos, calme, de bon ton et régulièrement dialogué, selon les règles de toute philosophie péripathétique, tourne autour d'un sujet que je perçois - à vrai dire - assez mal, mais qui doit être quelque chose comme: le vide peut-il avoir une forme? Ou plus précisément: comment un trou parviendrait-il à être pointu? $\gg^{41}$ On peut dès lors injecter du double sens, cesser de vouloir faire régner l'ordre c'est-à-dire de faire du papier l'instrument d'un pouvoir: "Égaré de façon définitive dans le dédale interne des organes, où je me suis aventuré, trompé par les fistules et les anastomoses, pris au piège de mon désir trop fort d'y faire régner l'ordre, mon ordre, j'ai perdu....je ne sais quel espoir, déraisonnable. J'ai perdu... Chose dorénavant parmi les choses, l'informe, peu à peu, se referme sur moi. »42 À partir de là, c'est sur des ruines qu'on (s') écrit c'est-à-dire en entrant dans « le désastreux

37 A. Robbe-Grillet, Les Derniers jours de Corinthe, p. 76.

38 A. Robbe-Grillet, Les Derniers jours de Corinthe, p. 17. On lit quelques pages plus loin l'idée de « subversion autobiographique » (p. 86). Angélique (p. 67) vise expressément Philippe Lejeune qui compte sur la signification comme organisation linéaire et principielle du récit et dont le livre (Le Pacte autobiographique, Paris: Seuil, 1996, p. 7) rappelle ce genre qu'est « l'écriture du moi », ce que son objecteur s'emploie à détruire.

39 A. Robbe-Grillet, Angélique ou l'enchantement, p. 173.

40 J . Lacan, Le Séminaire. Livre VII: l'éthique de la psychanalyse, Paris: Seuil, 1986, p. 272.

${ }^{41}$ A. Robbe-Grillet, Angélique, p. 231. On lira cette singerie comme humanisant d'un « h » le « péripatétique » qui vise Aristote (le Péripatéticien: celui qui marche).

42 A. Robbe-Grillet, Angélique, p. 232. 
cimetière des identités et des uniformes ${ }^{43}$. La ruine de l'identité - gommée: « vacante »44 - est par principe celle de la personnalité sous l'espèce du double ou plus exactement de la dissociation: «Celle qui regarde trop longtemps, le miroir l'a dédoublée »45. C'est donc une perte qu'il faut accepter: «j'ai écrit là deux romans successifs où s'exprime une conscience enfermée dans son propre vide, bien qu'entièrement tournée vers le dehors, à commencer par ce Voyeur qui s'appelait d'abord la Vue, en hommage à Raymond Roussel, puis le Voyageur ${ }^{46}$. Ainsi la forteresse - vide - a-t-elle sauté et le forçat de l'écriture a-t-il eu raison de Vauban: « Où en suis-je, d'ailleurs, exactement? Toutes ces choses, perdues dans le dédale obscur de la mémoire, ne montrent-elles pas une tendance alarmante à la perte progressive d'identité? ${ }^{47}$ Non pas; l'absence de centre narratif mobilise seulement l'image d'un lui qui fut: «Personnage impersonnel aussi mobile que variable, à l'identité précaire, ouverte à tous les vents, sa psyché - fort loin de la plénitude tranquille qu'elle affichait autrefois - n'est plus faite à présent que de failles, d'absences et d'aporétiques contradictions en chaînes. \} ^ { 4 8 } \text { Tout cela est le contraire de mythifier, c'est l'envers du } décor, l'enfermement décrit enfin comme - du - dehors; sur les pas de Maurice Blanchot, avance la nouvelle écriture qui décompose le moi c'est-à-dire le sujet-un qu'assoit le mythe du double.

\section{LE MYTHE DE L'IDENTITÉ}

« Ce récit est une fiction, non un témoignage. » Ainsi prévient l’avant-propos de Dans le labyrinthe, comme pour distinguer la fiction moderne du mythe ancien; se rouvre là cette question de la vérité, de la véridicité, du dire - à fortiori du mensonger - qui est celle de l'histoire, petite ou grande, et de la mise en cause du mythe pour sa fonction d'endoctrinement. $\mathrm{Si}$ « toute vérité a une structure de fiction »49, la vérité du soi prend

${ }^{43}$ A. Robbe-Grillet, Les Derniers jours de Corinthe, p. 153.

${ }^{44}$ A. Robbe-Grillet, Les Derniers jours de Corinthe, p. 80.

45 A. Robbe-Grillet, Topologie d'une cité fantôme, p. 123.

46 A. Robbe-Grillet, Les Derniers jours de Corinthe, p. 74. Qu'on pense aux motifs communs avec le texte rousselien (l'oblique, la ligne et le rayon visuel, la mer, la plage, le verre, l'écriteau, la porte, etc.); qu'on lise ceci (R. Roussel, La Vue, J .-J . Pauvert, 1963, p. 144): « Derrière ce rideau, leur silhouette double / Est nuageuse, sans fin, confuse et trouble ». Qu'on relise aussi La Doublure où Roussel écrit le double sous toutes ses coutures (p. 8, 34, 43, 189) et où le moi est trouvé détestable par l'un des personnages (p. 93).

${ }^{47}$ A. Robbe-Grillet, Les Derniers jours de Corinthe, p. 208.

48 A. Robbe-Grillet, Les Derniers jours de Corinthe, p. 145. Une lecture qui s'alarme de cette absence rate le jeu et le plaisir du texte. Ainsi soulève-t-on au sujet de Corinthe, qu'on a voulu voir comme une figure du double paternel, « le problème que représente l'identité de ce même comte, avant de définir l'identité de son moi propre » (Duplications et duplicités, p. 46) et « le problème de la véritable identité » (op. cit., p. 51); ainsi déplore-t-on qu' « il n'y a donc plus véritablement d'identité entre auteur et narrateur » (ib., p. 45) selon un pacte que Robbe-Grillet, qui fait partie des « écrivains ludiques » de la mouvance néo-romanesque (R.-.M. Allemand \& Ch. Milat, Introd. à Balises pour leXXIe siècle, 2010, p. 41), a explicitement rompu.

49 J . Lacan, Le Séminaire. Livre VII, p. 21. 
alors un sacré coup: «J e ne crois pas à la Vérité »50. La relation démystifiée à la vérité fait aussitôt ricochet sur celle d'identité que le mythe sacralise et que l'écriture désacralise si l'on considère par ailleurs que la littérature « est faite pour décevoir toute identité et pour tromper la compréhension comme pouvoir d'identifier ${ }^{51}$.

Le miroir qui revient thématise l'identité comme mythe, le mythe de l'identité: « un objet en soi contradictoire: l'attentat suprême qui détruit en même temps sa propre inscription. Et la mer apparaît, ce double de moi-même qui efface la marque de mes pas »52. Le jeu intertextuel aocomplit une révolution mettant fin à cette souveraineté d'où tient le sujet c'est-à-dire l'unité; Un Régicide légitime ce geste d'écriture libératrice par l'image de l'épitaphe: « Ci-gît Red »53. Ce nom à la couleur symbolique, le rouge révolutionnaire qui veut faire échec au projet régiste est précisément celui d'un étudiant; l'inscription qui le contient se donne clairement comme l'anagramme de " régicide ». C'est bien l'effacement d'un espace - moïque, idéologique - auquel procède le roman pensé comme contre-mythe. Contre le vieux « discours réactionnaire »54 qui veut que ce soit une - et une seule - personne qui s'exprime quand on écrit, il s'agit de faire entendre que le texte n'est pas une projection - répétition - de soi. La volonté d'en finir avec un Ancien Régime dont 《 Retz »55 est le nom ambigu a par conséquent lieu sur l'échiquier du texte où l'auteur apparait comme une notion politique: « loi, foi, roi, joie, toi, moi, pas d'abstention; c'était un discours électoral. ${ }^{56}$ La mort de l'auteur est forcément l'instauration du désordre; l'ordre narratif a failli: « Encore une fois, il passa la revue des noms: Abba, Acer, Acimin... tous étaient en bon ordre, mais le sien manquait »57. Il est étrange que ce roi dont on nous parle soit par avance si effacé, sauf à concevoir le dernier des écrivains comme formule visant cet auteur-roi qui n'est pas à soi c'est-à-dire même pas son propre sujet; c'est donc ce très vieux projet que condamne le nouveau texte au jeu d'échec et de mat: « il fallait un coup qui mette vraiment en cause le corps de son auteur. »58

Dans le texte qui rompt avec le discours auctorial, le mythe du double se lit notamment d'une thématisation de l'espion et du contre-espion - de l'agent double - en fond de guerre dont l'identité fait figure de clef dans la mesure où elle est mise sur le même plan qu'une arme: "Il n'y a plus ni jumelles nocturnes, ni pistolet de précision, ni carte d'identité, ni pochette de cuir dur perforée d'un trou sanglant. »59. L'obsession du texte se

50 A. Robbe-Grillet, Le Miroir qui revient, p. 11.

${ }^{51}$ M. Blanchot, L'Entretien infini, Paris: NRF Gallimard, 1969, p. 595.

52 A. Robbe-Grillet, Le Miroir qui revient, p. 43.

53 A. Robbe-Grillet, Un Régicide, p. 58. Voir aussi Le Miroir qui revient (p. 166).

54 A. Robbe-Grillet, Le Miroir qui revient, p. 10.

55 On pense aussi bien au cardinal de Retz qu'à « Gilles de Retz », ce personnage visé par la Topologie d'une cité fantôme et qui figure les meurtres perpétrés sur des enfants etc.

56 A. Robbe-Grillet, Un Régicide, p. 179.

${ }^{57}$ A. Robbe-Grillet, Un Régicide, p. 207.

58 A. Robbe-Grillet, Un Régicide, p. 158.

${ }^{59}$ A. Robbe-Grillet, La Reprise, p. 46. 
traduit par celle de lignes à passer: « J'étais une zone démilitarisée, un observateur solitaire et sans mandat, oublié dans une ville ouverte... »60 L'identité introuvable jalonne un roman de l'absence et du non-souci de soi. Les lignes de partage tombent; les lignes de fraction, de démarcation, bougent suffisamment pour que l'on s'interdise de les passer. Les zones frontalières s'estompent, comme les personnages mobilisés par le roman qui partent aux lignes de front pour franchir une grille et passer, dépasser pour trépasser. Le mythe de l'identité est dénoncé nonobstant la répétition d'un ordre détestable c'est-à-dire la reprise d'une mise en ordre ou d'un classement simplet et contraint; tel est l'effet de personnage, un effet de surface ou de miroir qui renvoie quelque chose de problématique: « un unique individu doublé de son reflet dans un miroir »61. Qu'importe qui soit qui? La question qui? cède à la question où? Dans le texte robbe-grilletien, il y a quelque chose de drôle à vouloir démultiplier le double pour, à juste titre, rendre l'identité douteuse: « Si l'on est seule, il faut faire semblant d'être deux. Si l'on est deux, faire semblant d'être trois. »

La critique de la notion d'auteur, Alain Robbe-Grillet la reprend à J ean Ricardou pour lequel cette notion ressortit à la fiction d'un hors texte selon le dogme de la représentation et, partant, celui d'une première personne illustrant la notion d'identité; ainsi visait-il le projet autobiographique se définissant par un contrat de lecture qui soit « un contrat d'identité »62. Si donc « au centre de la littérature, l'écriture est la contestation même »63, alors il faut reconnaître que c'est principalement celle de cette unité du personnage ou de la personne et que l'identité ressortit à la fabrique textuelle; le scripteur, cet opérateur changeant, a bien pris la place de l'auteur. Le texte robbe-grilletien ne cesse d'en perpétrer le meurtre; Corinthe, alias l'auteur c'est-à-dire le double énigmatique de Robbe-Grillet, meurt à plusieurs reprises. Telle est la fin d'un mythe dans un texte où la glace coupante fait lire la multiplicité au lieu de l'unité ou de la globalité selon une vue gestalltiste de la perception. Aucune prise n'est plus possible, dans un sens comme dans l'autre c'est-à-dire adverse ou non; rien n'est à réconcilier ni à concilier. Le nouveau roman dénonce la falsification réaliste: « De l'auteur lui-même, nous savons peu de chose »64, ajoutant que sa « véritable identité, déjà, est problématique. »65

60 A. Robbe-Grillet, Le Miroir qui revient, p. 136. Voir aussi Un Régicide, p. 78. La question de la différence des sexes occupe forcément le projet d'une nouvelle écriture, de même que celle de la droite et de la gauche; ainsi le brouillage de « la signification des lignes » (Le Voyeur, p. 168) met-il en crise cette différence du mythe œedipien en évoquant une « très jeune femme sans identité » (p. 224) et en alléguant « une distance imprécisable de ce mur, la ligne déterminée par l’homme et la femme ».

61 A. Robbe-Grillet, Projet pour une révolution à New York, p. 121. On rappellera ce « rapport où tout est double, contradictoire et fuyant » (Le Miroir qui revient, p. 41).

$62 \mathrm{Ph}$. Lejeune, Le Pacte autobiographique, p. 33.

63 J. Ricardou, Pour une théorie du nouveau roman, Paris: Seuil, 1971, p. 32. Pour cette référence à l'évacuation de la notion d'auteur, A. Robbe-Grillet, "Pourquoi j’aime Roland Barthes », in Prétexte: Roland Barthes, Paris: Ch. Bourgeois éditeur, 2003, p. 274 et p. 276.

${ }^{64}$ A. Robbe-Grillet, Djinn, p. 7.

${ }^{65}$ A. Robbe-Grillet, Djinn, p. 8. 
Une fois de plus, le héraut d'une nouvelle écriture rappelle sa proximité avec un projet philosophique d'ouverture lorsqu'il évoque le « sentiment d'extériorité [...] et presque d'exterritorialité »66. Plusieurs fois, Alain Robbe-Grillet invoque le philosophe du cinéma: « empruntant à Logique du sens de Gilles Deleuze sa représentation d'un fonctionnement structurel, je déployais un théorie des débris mobiles »67. Comme lui, il honnit la représentation et se positionne par un texte faisant figure d'anti-mythe autant que L'AntiEdipe; une lecture relève du reste la présence commune dans leurs textes respectifs de l'araignée et du fil (qui traverse les ensembles ou les plans) - « ténu »68 selon Cinéma 2 qu'on suit ou qu'on coupe, ou qu'on reprend. Les textes deleuzien et robbe-grilletien se recoupent en divers points, inspirés par la figure de la ligne. Dans le texte littéraire, la ligne d'écriture révolutionne les plans; des lignes - droites, horizontales, verticales, obliques, ligne du regard, de vue, de visée, d'inquiétude et de fureur, etc. - traversent en tous sens l'espace. Cette histoire de « ligne droite »69 file depuis le texte blanchotien qui médite sur l'égarement et consacre quelques pages à Robbe-Grillet à propos de l'absence et du manque organisant un autre centre: « comment y aurait-il encore là un nom et une identité? C'est sans nom, sans visage; c'est la pure présence anonyme. »70 Contrairement au philosophe qui cartographie, le nouveau romancier géométrise et pousse sans doute plus loin l'écriture en avançant la ligne: « L'homme y est présent à chaque page, à chaque ligne, à chaque mot. Même si l'on y trouve beaucoup d'objets, et décrits avec minutie, il y a toujours et d'abord le regard qui les voit, la pensée qui les revoit, la passion qui les déforme. ${ }^{71}$ Mais dans les deux cas, le texte détruit le mythe du sujet c'est-à-dire cette concrétion fascisante à laquelle il prête en tant que doté d'une identité, cette arme redoutable de toute propagande. Avec Pour un nouveau roman, tout se passe comme si la philosophie se déterritorialisait et qu'elle se faisait hors du champ clos de la rationalité euclidienne; qu'on l'entende: « La fiction romanesque, c'est déjà comme le devenir monde de la philosophie. ${ }^{72}$

${ }^{66}$ A. Robbe-Grillet, Le Miroir qui revient, p. 149.

${ }^{67}$ A. Robbe-Grillet, Les Derniers jours de Corinthe, p. 153. Deleuze est également mentionné aux pages 10 et 17 du même livre.

68 G. Deleuze, Cinéma 1, Paris: Minuit, 1983, p. 31. Le philosophe partage avec l'écrivain un intérêt pour le labyrinthe et l'araignée; et tous deux contribuent au projet de « la ruine du principe identitaire » (J . Poirer: « Perdre le fil: labyrinthes de la littérature française moderne », Amaltea, Vol. 1, 2009, p. 215).

69 G. Deleuze, Cinéma 2, p. 171: « la ligne droite, [...], comme labyrinthe du temps, est aussi la ligne qui bifurque et ne cesse de bifurquer, passant par des présents incompossibles, revenant sur des passés nonnécessairement vrais. » Chez Robbe-Grillet, aller à la ligne et se conformer à la droiture du script permet d'éprouver cette courbure par laquelle rien n'est plus comme avant c'est-à-dire figé ou pétrifié dans une quelconque identité; chez lui, la ligne est (une) coupe et le plan y est coupé alors qu'il est une coupe à lui tout seul chez Deleuze.

70 M. Blanchot, « La clarté romanesque », in Le Livre à venir, NRF Gallimard, 1959, note 1, p. 200.

${ }^{71}$ A. Robbe-Grillet, Pour un nouveau roman, p. 116. La théorie dit là que les lignes s'accumulent au fur et à mesure que les images s'évanouissent.

${ }^{72}$ A. Robbe-Grillet, Le Miroir qui revient, p. 69. 


\section{CONCLUSION: QUITTE}

La mise à jour du mythe, c'est-à-dire de l'image dans sa dimension captatrice idéologique - comme s'il n'y avait rien à en dire ni à redire, nous rend alors quitte par une retraversée du double qui est une traversée du miroir; nous nous en libérons peut-être, avant qu'en retombent des formes renouvelées. La quiétude de ne plus être dedans l'image - rend alors simple la réponse à la question textuelle du jeu: quitte ou double? Quitte, assurément. Le texte robbe-grilletien est doublement ludique; il est un jeu qui ne se prête pas à une lecture référenciante, il est un je qui ne se prête pas à une lecture unifiante. Je n'est donc pas un autre, car il est tout autre; son pluriel est celui du double. Aussi, soutenir que « c'est une parole qui ne parle de rien »73 est à la fois ne pas contrevenir à ce texte qui s'est débarrassé du référent et ne pas vouloir se tenir à lui. Le jeu textuel, c'est l'espace c'est-à-dire tout l'espace qu'il y a à écrire ou à inventer; le jeu est détente, entre ce qui est donné ou imposé de chaque côté ou de toutes parts. À suivre les indications d'un texte n'en finissant pas de composer et recomposer ses motifs, oui nous sommes enfin quittes. Parce que le double n'est pas la reprise comme Alain Robbe-Grillet avait insisté dans son entretien avec Benoît Peeters, son mythe est celui de l'identité; la ruine du vieux principe d'identité passe par la traversée d'un miroir dont on ne revient pas: « Mais voici, maintenant, que se distinguent mieux les contours et les surfaces: c'est moi, tout simplement. J e suis au-delà des parois en verre de la cage, de l'autre côté du miroir. »74 Cette traversée équivaut à coup sûr à la mise en pièce de l'identité vue aussi comme l'unité garantie par un papier non déchiré; le nouvel autobiographe le déclarait: « je ne suis pas d'une pièce ${ }^{75}$. Pour nous, il est quitte; et avec lui le texte qui nous inscrit comme ses lecteurs.

De quoi sommes-nous donc quitte(s) depuis Alain Robbe-Grillet? De l'antique logique qui définit l'ordre poétique comme principe tyrannique du discours. Dans le texte moderne, le mythe du double opère sans contredit une destruction de cette parole censée tenir d'elle-même et enfermer dans la représentation; dans sa finalité anti-narrative, l'écriture met fin au mythe comme à un type de cohérence; elle vaut ainsi dans son application comme prise à revers d'« un film de forme traditionnelle, je veux dire un récit linéaire aux enchaînements “logiques”. »76

${ }^{73}$ F. J. Matthews (pseudonyme d’A. Robbe-Grillet), « Un écrivain non réconcilié », Suite à La maison de rendez-vous, p. 182.

${ }^{74}$ A. Robbe-Grillet, Topologie d'une cité fantôme, p. 124.

75 A. Robbe-Grillet, Entretien avec J . Montalbetti, Magazine littéraire (janvier 1985), p. 93.

${ }^{76}$ A. Robbe-Grillet, Introduction à L'année dernière à Marienbad, p. 12. La fin de La Jalousie (p. 216) ne s'embarrasse plus du très ancien principe de contradiction, et partant du principe d'identité:

« Le personnage principal du livre est un fonctionnaire des douanes. Le personnage n'est pas un fonctionnaire, mais un employé supérieur d'une vieille compagnie commerciale. Les affaires de cette compagnie sont mauvaises, elles évoluent rapidement vers l'escroquerie. Les affaires de la compagnie sont très bonnes. Le personnage principal - apprend-on - est malhonnête. Il est honnête, il essaie de rétablir une situation 
L'interviewé avait rappelé qu'on crée de l'énergie avec des contradictions. Cette logique renversante, ce sont les motifs de lítinéraire et de ses bifurcations - de la traversée et de la retraversée du labyrinthe - qui l'inscrivent dans le nouveau corps du texte; effacer les traces, se démarquer, se perdre, sont ses dimensions ultimes: « l'instinct de conservation me fait changer de route une fois de plus. " ${ }^{77}$ Bifurquer, c'est aussi doubler c'est-à-dire aller en parallèle. Paradoxalement et pour la vie, le choix robbe-grilletien s'avère frontal: « Comment se décider? En tirant au sort à pile ou face? Face. » ${ }^{78}$

\section{BIBLIOGRAPHIE}

Allemand, Roger-Michel (1991). Duplications et duplicité dans les “Romanesques" d'Alain Robbe-Grillet. Paris, Lettres modernes, Archives des lettres modernes, 250.

—. et Milat, Christian (2010) (sous la direction de). Alain Robbe-Grillet. Balises pour le XXIe siècle. Les Presses de l'Université d'Ottawa, Les Presses de la Sorbonne Nouvelle.

Blanchot, Maurice (1959). Le Livreà venir. Paris: NRF Gallimard.

—. (1969). LEntretien infini. Paris: NRF Gallimard.

Bouveresse, J acques (1987). Le mythe de l'intériorité. Expérience, signification et langage privé chez Wittgenstein. Paris: Minuit, Coll. "Critique".

Deleuze, Gilles (1989). Différence et répétition. Paris: PUF (1ère éd. 1968), Coll. “La bibliothèque de philosophie contemporaine".

—. (1983). Cinéma 1. L'image-mouvement. Paris: Minuit, Coll. "Critique".

—. (1985). Cinéma 2. L'image temps. Paris: Minuit, Coll. "Critique”.

Dossier de Presse Les Gommes et Le Voyeur d'Alain Robbe-Grillet (1953-1956). Textes réunis et présentés par Emmanuelle Lambert (2005). Les Éditions de l'IMEC, 10/ 18.

Hadjji, Jalila (2009). La Quête épistémologique du Nouveau Roman, les objets. Paris: Publibook.

compromise par son prédécesseur, mort dans un accident de voiture. Mais il n’a pas eu de prédécesseur, car la compagnie est de fondation toute récente; et ce n'était pas un accident. Il est d'ailleurs question d'un navire (un grand navire blanc) et non de voiture. »

Dans Djinn (p. 44), le théoricien moderne récrit la logique aristotélicienne c'est-à-dire la logique philosophique antique:

« En classe de logique, nous faisons cette année des exercices de mensonge au second degré. Nous étudions aussi le mensonge au premier degré et à deux inconnues. Et quelquefois, nous mentons à plusieurs voix, c'est très excitant. Dans la classe supérieure, elles font le mensonge du second degré à deux inconnues et le mensonge au troisième degré. »

${ }^{77}$ A. Robbe-Grillet, Un Régicide, p. 221.

${ }^{78}$ A. Robbe-Grillet, Un Régicide, pp. 148-9. 
Lacan, Jacques (1978). Le Séminaire. Livre II: le moi dans la théorie de Freud et dans la technique de la psychanalyse (1954-1955). Texte établi par Jacques-Alain Miller. Paris: Seuil, Coll. "Le champ freudien”.

—. (1986). Le Séminaire. Livre VII: l'éthique de la psychanalyse (1959-1960). Texte établi par J acques-Alain Miller. Paris: Seuil, Coll. "Le champ freudien".

Lambert, Emmanuelle (2009). Mon grand écrivain. Les Impressions nouvelles, Coll. “Traverses".

Laouyen, Mounir (2010). L'autobiographie à l'ère du soupçon: Barthes, Sarraute, et Robbe-Grillet. Clermont-Ferrand: Université Blaise Pascal II.

Lejeune, Philippe (1996). Le Pacte autobiographique. Paris: Seuil, Coll. “Essais”, lère édition 1975.

Loraux, Nicole (1996). Né de la terre. Mythe et politique à Athènes. Paris: Seuil, Coll. "La librairie du XXIe siècle".

Mancas, Magdalena Silvia (2010). Pour une esthétique du mensonge: nouvelle autobiographie et postmodernisme. Frankfurt am Main, Bruxelles: Peter Lang, Coll. "Langue et littérature françaises".

Poirier, J acques (2009). « Perdre le fil: labyrinthes de la littérature française moderne ». Amaltea, Vol. 1. <http:// www.ucm.es/ info/amaltea/ revista/ num1/ poirier.pdf>.

Prédal, René (2005). Robbe-Grillet cinéaste. Caen: Presses universitaires de Caen.

Ricardou, J ean (1971). Pour une théorie du nouveau roman. Paris: Seuil, Coll. "Tel Quel”.

—. (1976). Robbe-Grillet, Colloque de Cerisy: Analyse, théorie 1. Roman/Cinéma, 10/ 18: 10-33.

Robbe-Grillet, Alain (1953). Les Gommes. Paris: Minuit.

—. (1955). Le Voyeur. Paris: Minuit.

_. (1957). La J alousie. Paris: Minuit.

—. (1959). Dans le labyrinthe. Paris: Minuit.

—. (1961). L’année dernière à Marienbad, Ciné-roman, Illustré de 48 photographies extraites du film réalisé par Alain Resnais. Paris: Minuit.

—. (1962). Instantanés, nouvelles. Paris: Minuit.

—. (1963). LTmmortelle, Ciné-roman, Illustré de 40 photographies extraites du film. Paris: Minuit.

—. (1963). Pour un nouveau roman. Paris: Minuit, Coll. "Critique”.

—. (1965). La Maison de rendez-vous, Suivi de Un écrivain non réconcilié par Franklin J . Matthews. Paris: Minuit.

— (1970). Projet pour une révolution à New York. Paris: Minuit.

—. (1974). Glissements progressifs du plaisir. Paris: Minuit. 
—. (1976). Topologie d'une cité fantôme. Paris: Minuit.

—. (1977). « Pourquoi j'aime Roland Barthes ». Prétexte: Roland Barthes, Colloque de Cerisy (sous la dir. d'Antoine Compagnon). Paris: Ch. Bourgeois éditeur, 2003.

—. (1978). Un Régicide. Paris: Minuit.

—. (1981). Djinn. Un trou entre les pavés disjoints. Paris: Minuit.

—. (1984). Le Miroir qui revient. Paris: Minuit.

—. (1985). « Alain Robbe-Grillet autobiographe ». Entretien, Magazine littéraire, Propos recueillis par J ean Montalbetti: 88-93.

—. (1987). Angélique ou l'enchantement. Paris: Minuit.

—. (1994). Les Derniers jours de Corinthe. Paris: Minuit.

—. (2001). La Reprise. Paris: Minuit.

—. (2001). Le Voyageur. Textes, causeries et entretiens (1947-2001). Choisis et présentés par Olivier Corpet avec la collaboration d'Emmanuelle Lambert. Paris: Christian Bourgeois.

- (2001). Entretiens avec Benoît Peeters. Les Piérides: Les impressions nouvelles/ IMEC (double DVD vidéo), J eux de mémoire.

Roussel, Raymond (1963). La Vue. J ean-J acques Pauvert.

—. (1963). La Doublure. J ean-J acques Pauvert.

Roussel-Meyer, Maryse (2011). La fragmentation dans le roman: Louis-Ferdinand Céline,

Robert Pinget, Alain Robbe-Grillet. Clermont-Ferrand: Presses universitaires Blaise Pascal.

Simonin, Anne (2008). Les Éditions de Minuit 1942-1955. Le devoir d'insoumission, Nouvelle édition augmentée avec des annexes sur le Nouveau Roman et la Guerre d'Algérie. Mimec-éditeur, Coll. “L'édition contemporaine”.

Wagner, Frank, Dugast-Portes, Francine (2010). [sous la direction de], Lectures de RobbeGrillet, Les Gommes, La J alousie.Rennes: Presses Universitaires de Rennes (PUR), Coll. “Didact français4. 